Pacific Journal of Mathematic 


\title{
ABSOLUTE SUMMABILITY OF WALSH-FOURIER SERIES
}

\author{
N. R. LADHAWALA
}

We prove that for all $f \in \mathscr{H}^{1}, \sum_{k=1}^{\infty}(1 / k)|\hat{f}(k)| \leq K\|f\|_{\mathscr{C}_{1}}$, where $\mathscr{H}^{1}$ is the Walsh function analogue of the classical Hardy-space and $\hat{f}(k)$ is the $k^{\text {th }}$ Walsh-Fourier coefficient of $f$. We obtain this as a consequence of its dual result: given a sequence $\left\{a_{k}\right\}$ of numbers such that $a_{k}=O(1 / k)$, there exists a function $h \in \mathbf{B M O}$ with $\hat{h}(k)=a_{k}$.

We study the relation between our results and the theory of differentiation on the Walsh group, developed by Butzer and Wagner.

Introduction. We are interested in various properties of Walsh-Fourier series. $w_{k}(\cdot)$ will denote the $k^{\text {th }}$ Walsh function in the Paley-enumeration and $\hat{f}(k)$ will be the corresponding WalshFourier coefficient of $f \in L^{1} . \mathscr{X}^{1}$ and BMO will denote the Walsh function analogues of the classical Hardy space and the functions of bounded mean oscillation, respectively (see [3], pp. 3-4; also refer to the section on "Preliminaries", in this paper).

Our principal result is

THEOREM 1. There exists a constant $K>0$ such that

$$
\sum_{k=1}^{\infty}(1 / k)|\hat{f}(k)| \leqq K\|f\|_{\mathscr{*} 1},
$$

for all $f \in \mathscr{X}^{1}$.

Our proof of Theorem 1 does not follow the lines of its trigonometric analogue (see [5], pp. 286-287). We use the fact that Theorem 1 is equivalent to

THEOREM 2. Given a sequence $\left\{a_{k}\right\}$ of numbers such that $a_{k}=O(1 / k)$, there exists a function $h$ in BMO such that $\hat{h}(k)=a_{k}$ for all $k$.

We give a direct proof of Theorem 2 .

Theorem 2, combined with a result of Fine [2] gives Lip $\left(1, L^{1}\right) \subseteq$ BMO. However, $\operatorname{Lip}\left(1, L^{1}\right) \not \subset L^{\infty}$, in contrast with the trigonometric case where $\operatorname{Lip}\left(1, L^{1}\right)=B V \subseteq L^{\infty}$ (see [5], p. 180). Theorem 2 also has connections with the Butzer-Wagner theory of differentiation on the Walsh-group (see [1]). The antidifferentiation kernel $W(x) \sim 1+$ $\sum_{k=1}^{\infty}(1 / k) w_{k}(x)$ was shown by Butzer-Wagner to be in $\operatorname{Lip}\left(1, L^{1}\right)$. 
$W$ is thus a function in BMO, but $W$ is not bounded. Hence we know that if both $f$ and $D^{[1]} f$-the Butzer-Wagner derivative of $f$-are in $L^{1}$ then $f$ must be in BMO. We give an example of an $f$ in $L^{1}$ with $D^{[1]} f$ in $L^{1}$ but $f$ not bounded.

We have that $W \in \operatorname{Lip}\left(1 / p, L^{p}\right)$, for $1 \leqq p<\infty$, which gives: if both $f$ and $D^{[1]} f$ are in $L^{p}, 1<p<\infty$, then $f$ is in $\operatorname{Lip}\left(1 / p^{\prime}, C(G)\right)$ and the Walsh-Fourier series of $f$ converges absolutely.

Theorem 1 can be restated in the context of the Butzer-Wagner theory as: if $f$ and $D^{[1]} f$ are in $\mathscr{H}^{1}$ then $\sum_{k=1}^{\infty}|\hat{f}(k)|<\infty$. Equivalently, the Walsh-Fourier series of the 'indefinite integral' of any $f$ in $\mathscr{H}^{1}$ converges absolutely.

I am grateful to R. A. Hunt and R. C. Penney for their help and encouragement.

Preliminaries. $G=\Pi \boldsymbol{Z}_{2}$, the countable product of infinitely many copies of $\boldsymbol{Z}_{2}$, is called the Walsh-group. Addition in $G$, defined termwise modulo 2, is denoted by + . For a fixed $x=\left(x_{k}\right) \in G$, the sets $V_{0}(x)=G$,

$$
V_{n}(x)=\left\{\left(x_{1}, x_{2}, \cdots, x_{n}, z_{n+1}, z_{n+2}, \cdots\right) \in G\right\}, n \geqq 1,
$$

define a neighbourhood system at $x$ and the topology thus induced on $G$, makes it a compact, abelian group.

The Haar-measure ' $d x$ ' on $G$ is normalized so that $\int_{G} d x=1$. The character group $\hat{G}$ of $G$ is the set of all continuous, nonzero functions $\chi$ on $G$, satisfying

$$
\chi(x+y)=\chi(x) \chi(y), \forall x, y \in G,
$$

endowed with the compact-open topology. Fine [2] has shown that these functions are given by

$$
w_{n}(x)=\prod_{k=0}^{\infty}\left[r_{k}(x)\right]^{\varepsilon_{k}},
$$

where $r_{k}(x)=(-1)^{x} k+1$ and $n=\sum_{j=0}^{\infty} \varepsilon_{j} 2^{j}$ is the unique binary expansion of the integer $n \geqq 0 . \quad r_{k}$ 's are called the Rademacher functions and $w_{j}$ 's the Walsh-functions (in Paley's enumeration). The system $\left\{w_{j}\right\}$ is closed under pointwise multiplication; more precisely, $w_{n} \cdot w_{m}=w_{n+m}$, where for $n=\sum_{j=0}^{\infty} \varepsilon_{j} 2^{j}$, and $m=\sum_{j=0}^{\infty} \eta_{j} 2^{j}, \varepsilon_{j}, \eta_{j} \in$ $\{0,1\}$, we have $n+m=\sum_{j=0}^{\infty}\left|\varepsilon_{j}-\eta_{j}\right| 2^{j}$.

For $m \geqq 1$, the $m^{\text {th }}$ Dirichlet kernel is defined as:

$$
D_{m}(x)=\sum_{k=0}^{m-1} w_{k}(x) \text {. }
$$

For $m=2^{n}$, 


$$
D_{2^{n}}(x)=\left\{\begin{array}{rll}
2^{n} & \text { if } & x \in V_{n}(0) \\
0 & \text { if } & x \notin V_{n}(0)
\end{array}\right.
$$

For $f, g \in L^{1}$,

$$
(f * g)(x)=\int_{G} f(y) g(x+y) d y .
$$

$\hat{f}(k)=\int_{G} f(x) w_{k}(x) d x$ denotes the $k^{\text {th }}$ Walsh-Fourier coefficient and

$$
\left(S_{n} f\right)(x)=\sum_{k=0}^{n-1} \hat{f}(k) w_{k}(x)=\left(f * D_{n}\right)(x)
$$

is the $n^{\text {th }}$ partial sum of the Walsh-Fourier series of $f$. Thus,

$$
\left(S_{2^{m}} f\right)(x)=2^{m} \int_{V_{m}(x)} f(t) d t .
$$

Moreover, $(f * g)^{\wedge}(k)=\hat{f}(k) \hat{g}(k), \quad \forall k \geqq 0$ and $f, g \in L^{1}$. Henceforth, all functions $f$ are assumed to satisfy $\int_{G} f(x) d x=\hat{f}(0)=0$.

$L^{p}, 1 \leqq p \leqq \infty$ denote the usual Lebesgue spaces on $G ; C(G)$ is the space of continuous functions on $G$.

BMO is defined to be the space of all functions $f$ such that $\sup _{n \geqq 1}\left\|S_{2^{n}}\left[f-S_{2^{n-1}} f\right]^{2}\right\|_{\infty}<\infty$. $\mathscr{H}^{1}$ is the space of those functions $f$ for which $S f=\left(\sum_{n=1}^{\infty}\left[S_{2^{n}} f-S_{2^{n-1}} f\right]^{2}\right)^{1 / 2} \in L^{1}$. Moreover, $\|f\|_{\mathscr{F}^{1}}=$ $\|S f\|_{L^{1}}$ (see [3]).

For $h=\left(h_{n}\right) \in G$, let $\lambda(h)=\sum_{n=1}^{\infty} h_{n} \cdot 2^{-n}$, and $\operatorname{Lip}\left(\alpha, L^{p}\right)=\{f \in$ $\left.L^{p}:\|f(\cdot)-f(\cdot+h)\|_{L^{p}}=O\left[\lambda(h)^{\alpha}\right]\right\}, 1 \leqq p<\infty, \alpha>0$.

For $p=\infty$, we replace $L^{\infty}$ by $C(G)$. If

$$
\omega_{p}(f ; \delta)=\sup _{\lambda(h) \leqq \delta}\|f(\cdot)-f(\cdot+h)\|_{L^{p}},
$$

then $f \in \operatorname{Lip}\left(\alpha, L^{p}\right) \Leftrightarrow \omega_{p}(f ; \delta)=O\left(\delta^{\alpha}\right) \Leftrightarrow \omega_{p}\left(f ; 2^{-n}\right)=O\left(2^{-n \alpha}\right)$.

Let $X$ denote $L^{p}, 1 \leqq p<\infty$, or $C(G)$.

Define $e_{j}=\left\{x_{s}^{j}\right\}$ where $x_{s}^{j}=\delta_{j s}$. For an $f \in X$, if there exists a $g \in X$ such that $\lim _{m \rightarrow \infty}\left\|1 / 2 \sum_{j=0}^{m} 2^{j}\left[f(\cdot)-f\left(\cdot+e_{j+1}\right)\right]-g(\cdot)\right\|_{X}=0$, then $f$ is said to be differentiable in $X$ (see [1]). $g$ is called the derivative of $f$ and we write $D^{[1]} f=g$. Differentiable functions in $X$ are completely characterized by the Theorem (see [1]):

For $f \in X$, the following are equivalent:

(1) $D^{[1]} f=g$ exists.

(2) There is a $g \in X$ such that $k \hat{f}(k)=\hat{g}(k), \forall k$.

(3) There is a $g \in X$ such that $f=W * g$ where $W(x) \sim 1+\sum_{k=1}^{\infty}(1 / k) w_{k}(x)$.

Proof of Theorem 2. Since $a_{k}=O(1 / k)$, say $\left|a_{k}\right| \leqq M_{1}(1 / k)$, 
$\forall k \geqq 1, \sum_{k=1}^{\infty} a_{k} w_{k}(x)$ defines a function $h \in L^{2}$ such that $\hat{h}(k)=a_{k}$ for $k \geqq 1$ and $\hat{h}(0)=0$. Let us put, $D_{n}(h, \nu)(t)=S_{2^{n}}\left\{S_{2^{2}} h(\cdot) S_{2^{\nu}=1} h(\cdot)\right\}^{2}(t)$. Then to prove $h \in \mathrm{BMO}$, it suffices to show that (see [3]), $\exists M>$ 0 Э $\left\|\sum_{\nu=n}^{\infty} D_{n}(h, \nu)(t)\right\|_{\left(L^{\infty}, d t\right)} \leqq M, \forall n \geqq 1$.

Now

$$
\begin{aligned}
\left\{S_{2^{\nu}}\right. & \left.h(\cdot)-S_{2^{\nu-1}} h(\cdot)\right\}^{2} \\
& =\left\{\sum_{k=2^{\nu-1}}^{2^{\nu}-1} a_{k} w_{k}(\cdot)\right\}^{2} \\
& =\sum_{k=2^{\nu-1}}^{2^{\nu}-1} a_{k}^{2}+2 \sum_{k=2^{\nu-1}+1}^{2^{\nu}-1} \sum_{l=2^{\nu-1}}^{k-1} a_{k} \cdot a_{l} \cdot w_{k+l}(\cdot) .
\end{aligned}
$$

Also, for any $t \in G$ and $n$ fixed,

$$
S_{2}{ }^{n}\left(w_{j}\right)(t)= \pm \chi_{n}(j)=\left\{\begin{array}{cl} 
\pm 1 & \text { if } 0 \leqq j<2^{n} \\
0 & \text { otherwise }
\end{array}\right.
$$

Since $S_{2}{ }^{n}\left(w_{k+l}\right)(t)= \pm \chi_{n}(k+l)$, we have for $\nu \geqq n$

$$
\begin{aligned}
& D_{n}(h, \nu)(t) \\
& \quad=\sum_{k=2^{\nu-1}}^{2^{\nu}-1} a_{k}^{2}+2 \sum_{k=2^{\nu-1}+1}^{2^{\nu}-1} \sum_{l=2^{\nu-1}}^{k-1} \pm a_{k} \cdot a_{l} \cdot \chi_{n}(k+l) .
\end{aligned}
$$

Thus

$$
\begin{aligned}
& \left|\sum_{\nu=n}^{\infty} D_{n}(h, \nu)(t)\right| \\
& \quad \leqq M_{1}^{2}\left[\sum_{k=1}^{\infty} \frac{1}{k^{2}}+2 \sum_{\nu=n}^{\infty} \sum_{k=2^{\nu-1}+1}^{2^{\nu}-1} \sum_{l=2^{\nu-1}}^{k-1} \frac{1}{k} \cdot \frac{1}{l} \cdot\left|\chi_{n}(k+l)\right|\right] .
\end{aligned}
$$

Note that, $\left|\chi_{n}(k+l)\right|=1$ for $0 \leqq k+l<2^{n}$ and 0 otherwise. For a fixed $k, k+l<2^{n}$ iff the dyadic expansions of $k$ and $l$ agree at and after the $n^{\text {th }}$ stage. Thus, there are exactly $2^{n}$ values of $l$ for which $\left|\chi_{n}(k+l)\right|=1$, if $k$ is fixed. Therefore

$$
\begin{aligned}
\sum_{\nu=n}^{\infty}\left\{\sum_{k=2^{\nu-1}+1}^{2^{\nu}-1} \frac{1}{k} \sum_{l=2^{\nu-1}}^{k-1} \frac{1}{l} \cdot\left|\chi_{n}(k+l)\right|\right\} \\
\quad<\sum_{\nu=n}^{\infty}\left\{\sum_{k=2^{\nu-1}+1}^{2^{\nu}-1} \frac{1}{k} \cdot \frac{2^{n}}{2^{\nu-1}}\right\}<\sum_{\nu=n}^{\infty} \frac{2^{n}}{2^{\nu-1}}=4 .
\end{aligned}
$$

Since $\sum_{k=1}^{\infty}\left(1 / k^{2}\right)<\infty, \exists M<\infty$ such that $\left|\sum_{\nu=n}^{\infty} D_{n}(h, \nu)(t)\right|<M$, i.e., $\left\|\sum_{\nu=n}^{\infty} D_{n}(h, \nu)(t)\right\|_{\left(L^{\infty}, d t\right)} \leqq M<\infty, \forall n \geqq 1$.

Corollary 1. Lip $\left(1, L^{1}\right) \cong \mathrm{BMO}$, but $\operatorname{Lip}\left(1, L^{1}\right) \cong L^{\infty}$.

Proof. Fine [2] had proved that, for each $f$ in $\operatorname{Lip}\left(1, L^{1}\right)$, $\hat{f}(k)=O(1 / k)$. So $f \in \mathrm{BMO}$ by Theorem 2 . 
Butzer and Wagner [1] have shown that $W(x) \sim 1+\sum_{k=1}^{\infty}(1 / k) w_{k}(x)$ is in $\operatorname{Lip}\left(1, L^{1}\right)$. But $W \notin L^{\infty}$ because $\left\{S_{2^{n}} g\right\}$ is uniformly bounded whenever $g \in L^{\infty} ; S_{2^{m}} W(x)=1+\sum_{k=1}^{2^{m}-1}(1 / k), \forall x \in V_{m}(0)$.

REMARK. The above corollary is in contrast with the trigonometric case. We know that $\operatorname{Lip}\left(1, L^{1}\right)=B V \cong L^{\infty}$ in the latter context [5, p. 180].

Proof of Theorem 1. Recall that $f \in \mathscr{H}^{1}$. We want to show that $\sum_{k=1}^{\infty}(1 / k)|\hat{f}(k)| \leqq K \cdot\|f\|_{\mathscr{F}^{1}}$, with $K$ independent of $f$.

Let us put $b_{k}=(\operatorname{sgn} \hat{f}(k)) / k, k \geqq 1, b_{0}=0$. Then by Theorem $2, \exists g \in \mathrm{BMO}$ such that $\widehat{g}(k)=b_{k}$. Therefore

$$
\begin{aligned}
\sum_{k=1}^{2^{N}-1}(1 / k)|\hat{f}(k)| & =\left(S_{2^{N}} g * S_{2^{N}} f\right)(0) \\
& =\int_{G}\left(S_{2^{N}} g\right)(y) \cdot\left(S_{2^{N}} f\right)(y) d y .
\end{aligned}
$$

But (see [3], p. 8) the last integral is majorized by

$$
\sqrt{2}\|g\|_{\text {вмо }}\|f\|_{\mathscr{C}^{1}} \text {. }
$$

Thus $\sum_{k=1}^{\infty}(1 / k)|\hat{f}(k)| \leqq \sqrt{2}\|g\|_{\text {Вмо }}\|f\|_{\mathscr{F}^{1} \text {. }} \quad$ Вy the proof of Theorem 2, $\|g\|_{\text {вмо }} \leqq \pi^{2} / 6+8$. Hence, there exists a constant $K>0$, independent of $f$, such that

$$
\sum_{k=1}^{\infty}|\hat{f}(k)|(1 / k) \leqq K\|f\|_{\varkappa^{1}} .
$$

REMARK. It can be easily shown that Theorem 1 implies Theorem 2.

Butzer and Wagner ([1]) introduced the notion of differentiation on the Walsh-group. They showed that $W(x) \sim 1+\sum_{k=1}^{\infty}(1 / k) w_{k}(x)$ is the 'antidifferentiation' kernel and $W$ belongs to $\operatorname{Lip}\left(1, L^{1}\right)$. In the proof of Corollary 1 , we have shown that $W \in \mathrm{BMO}$ but $W \notin L^{\infty}$. Since convolution of an $L^{1}$ function and a BMO function is again a BMO function, we obtain $f$ and $D^{[1]} f$ are in $L^{1} \Rightarrow f=W * D^{[1]} f$ is in BMO. Rubinshtein [4] has shown that $\sum_{n=1}^{\infty}(1 / n \log n) w_{n}(x)$ defines an unbounded $L^{1}$-function $g$, and that $\sum_{n=2}^{\infty}(1 / \log n) w_{n}(x) \sim h(x)$ is in $L^{1}$. Thus, we have $g$ and $h=D^{[1]} g$ both in $L^{1}$ but $g$ is not bounded. It is easy to prove that $W \in \operatorname{Lip}\left(1 / 2, L^{2}\right)$; then using interpolation and duality, we get $W \in \operatorname{Lip}\left(1 / p, L^{p}\right), 1 \leqq p<\infty$. By the characterization of differentiable functions in $L^{p}$ (see [1]), we then have that if $f$ and $D^{[1]} f$ are in $L^{p}$ for some $1<p<\infty$, then $f \in \operatorname{Lip}(1 / q, C(G))$, where $1 / p+1 / q=1$. This leads to the fact that the Walsh-Fourier series of such an $f$ converges absolutely. Theorem 
1 actually strengthens this result, as we see below.

The definition of derivative can be given for $\mathscr{H}^{1}$ as in [1]. A characterization similar to $(*)$ for differentiability in $\mathscr{H}^{1}$ remains true: $f \in \mathscr{H}^{1}$ is differentiable iff $\exists g \in \mathscr{H}^{1}$ such that $k \hat{f}(k)=\hat{g}(k), \forall k$.

Now, if $f$ is differentiable in $\mathscr{H}^{1}$, then

$$
\sum_{k=1}^{\infty}|\hat{f}(k)|=\sum_{k=1}^{\infty}(1 / k)|\hat{g}(k)|<\infty
$$

by Theorem 1 , because $g \in \mathscr{H}^{1}$; thus $f$ has an absolutely convergent Walsh-Fourier series. The same fact can be stated as: The WalshFourier series of the "indefinite integral" $W * g$ of any $g \in \mathscr{H}^{1}$, is absolutely convergent.

\section{REFERENCES}

1. P. L. Butzer and H. J. Wagner, Walsh-Fourier series and the concept of a derivative, Applicable Analysis, 3 (1973), 29-46.

2. N. J. Fine, On the Walsh functions, Trans. Amer. Math. Soc., 65 (1949), 372-414.

3. A. M. Garsia, Martingale Inequalities, Seminar Notes on Recent Progress, W. A. Benjamin, Inc., Reading, Mass., 1973.

4. A. I. Rubinshtein, Series with respect to the Walsh system with monotone coefficients, Matem. Zametki, 2, No. 3(1967), 279-288.

5. A. Zygmund, Trigonometric Series, 2nd ed. Vol. I, II, Cambridge University Press, Cambridge, 1959.

Received February 2, 1976.

Purdue University 


\section{PACIFIC JOURNAL OF MATHEMATICS}

\section{EDITORS}

RICHARD ARENS (Managing Editor) University of California

Los Angeles, California 90024

R. A. Beaumont

University of Washington

Seattle, Washington 98105
J. DUGUNDJI

Department of Mathematics University of Southern Californı

Los Angeles, California 90007

D. Gilbarg and J. Milgram

Stanford University

Stanford, California 94305

\section{ASSOCIATE EDITORS}
E. F. BECKENBACH
B. H. NeumanN
F. WOLF
K. YosHIDA

\section{SUPPORTING INSTITUTIONS}

UNIVERSITY OF BRITISH COLUMBIA

CALIFORNIA INSTITUTE OF TECHNOLOGY

UNIVERSITY OF CALIFORNIA

MONTANA STATE UNIVERSITY

UNIVERSITY OF NEVADA

NEW MEXICO STATE UNIVERSITY

OREGON STATE UNIVERSITY

UNIVERSITY OF OREGON

OSAKA UNIVERSITY
UNIVERSITY OF SOUTHERN CALIFORNIA

STANFORD UNIVERSITY

UNIVERSITY OF TOKYO

UNIVERSITY OF UTAH

WASHINGTON STATE UNIVERSITY

UNIVERSITY OF WASHINGTON

* * * *

AMERICAN MATHEMATICAL SOCIETY

NAVAL WEAPONS CENTER 


\section{Pacific Journal of Mathematics \\ Vol. 65, No. $1 \quad$ September, 1976}

David Lee Armacost, Compactly cogenerated LCA groups ............. 1

Sun Man Chang, On continuous image averaging of probability measures ...... 13

J. Chidambaraswamy, Generalized Dedekind $\psi$-functions with respect to a

polynomial. II................................... 19

Freddy Delbaen, The Dunford-Pettis property for certain uniform algebras ..... 29

Robert Benjamin Feinberg, Faithful distributive modules over incidence

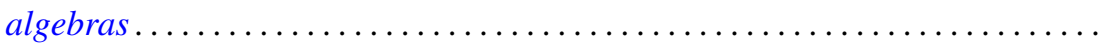

Paul Froeschl, Chained rings . . . . . . . . . . . . . . . . . . . .

John Brady Garnett and Anthony G. O'Farrell, Sobolev approximation by a sum

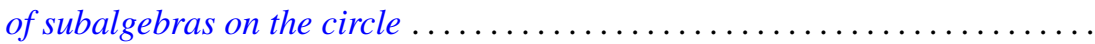

Hugh M. Hilden, José M. Montesinos and Thomas Lusk Thickstun, Closed

oriented 3-manifolds as 3-fold branched coverings of $S^{3}$ of special type.....

Atsushi Inoue, On a class of unbounded operator algebras ................

Peter Kleinschmidt, On facets with non-arbitrary shapes.

Narendrakumar Ramanlal Ladhawala, Absolute summability of Walsh-Fourier

series

Howard Wilson Lambert, Links which are unknottable by maps . . . . . . . . . . .

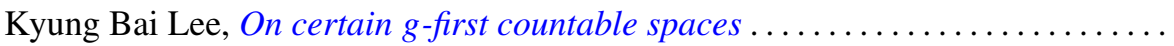

Richard Ira Loebl, A Hahn decomposition for linear maps .................

Moshe Marcus and Victor Julius Mizel, A characterization of functionals on $W_{1}^{p}$ possessing autonomous kernels. I . .

James Miller, Subordinating factor sequences and convex functions of several

variables.

Keith Pierce, Amalgamated sums of abelian l-groups ...

Jonathan Rosenberg, The $C^{*}$-algebras of some real and $p$-adic solvable

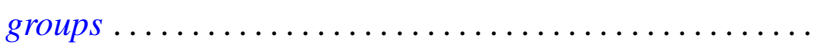

Hugo Rossi and Michele Vergne, Group representations on Hilbert spaces defined

in terms of $\partial_{b}$-cohomology on the Silov boundary of a Siegel domain . .

Mary Elizabeth Schaps, Nonsingular deformations of a determinantal

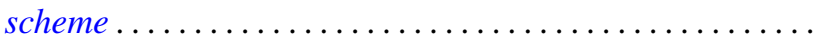

S. R. Singh, Some convergence properties of the Bubnov-Galerkin method...

Peggy Strait, Level crossing probabilities for a multi-parameter Brownian

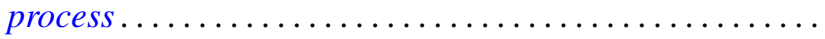

Robert M. Tardiff, Topologies for probabilistic metric spaces .

Benjamin Baxter Wells, Jr., Rearrangements of functions on the ring of integers of

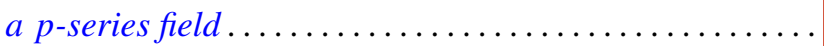

Robert Francis Wheeler, Well-behaved and totally bounded approximate identities for $C_{0}(X)$.

Delores Arletta Williams, Gauss sums and integral quadratic forms over local

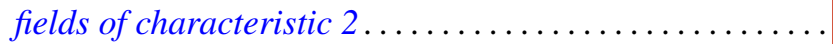

John Yuan, On the construction of one-parameter semigroups in topological 